\title{
LA FORMACIÓN EN CIUDADANÍA EN ESCENARIOS DE EDUCACIÓN INICIAL: UNA EXPERIENCIA CON MADRES COMUNITARIAS ${ }^{1}$
}

\author{
Citizenry Education within Early Childhood Scenarios: An Experience with Community \\ Mothers
}

A formação em cidadania em cenários de educação inicial: uma experiência com mães comunitárias

Arly Adriana Rodríguez Huesa (Colombia) Magíster en Educación

Universidad Santo Tomás

arlyrodriguez@usantotomas.edu.co

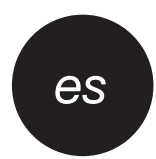

\section{RESUMEN}

El presente artículo analiza la formación ciudadana que brindan las madres comunitarias de Bogotá a niños y niñas de la primera infancia. Para ello, se organizaron grupos focales con 27 madres comunitarias que hacen parte del programa Técnico en Atención Integral a la Primera Infancia del Servicio Nacional de Aprendizaje (SENA). Se establecieron tres categorías para el análisis: participación, autonomía y pensamiento cuidante. Se realizó la visita al Hogar Comunitario Agrupado Domingo Savio de la localidad Santafé, y en este ejercicio emergieron tres nuevas categorías: ciudadanía en las madres comunitarias, juicio moral y resolución de conflictos, lo cual permitió ampliar el análisis. El estudio arroja entre sus resultados la importancia de concebir a los niños y las niñas de la primera infancia como interlocutores válidos y comprenderlos como sujetos políticos. Además, se observó que las educadoras, desde su condición de género, aportan elementos para comprender la ciudadanía de los más pequeños y que la formación que brinda el SENA ha tenido un impacto positivo en la resignificación de su ciudadanía, lo cual supone una afectación en la orientación ciudadana que promueven.

PALABRAS CLAVE: ciudadanía, primera infancia, madres comunitarias, participación, autonomía, pensamiento cuidante, resolución de conflictos, juicio moral.

\author{
Rita Flórez Romero (Colombia) \\ Magíster en Lingüística \\ Universidad Nacional de Colombia \\ rflorezr@unal.edu.co
}

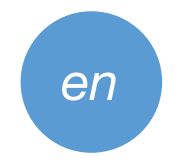

\section{ABSTRACT}

This article analyzes the education provided by community mothers in Bogotá to children in early childhood. In order to do this, we organized focal groups with $27 \mathrm{commu}-$ nity mothers that belong to the technical program in Comprehensive Assistance in Early Childhood belonging to the National Service of Learning (SENA for its name in Spanish.) We established three categories for the analysis: participation, autonomy, and watching thought. We visited the Community Home Agrupado Domingo Savio located in Santafé, and from this exercise, we obtained three new categories: community mothers' citizenry, moral judgement, and conflict resolution, which enabled enhancing the analysis. Among the results, we include the importance of considering these children as valid interlocutors and understanding them as political subjects. We also observed that the teachers, due to their gender, bring elements to understand the citizenry of the youngest children and that the training offered by SENA has a positive impact on the redefinition of their citizenry, which represents an influence on the civic education promoted.

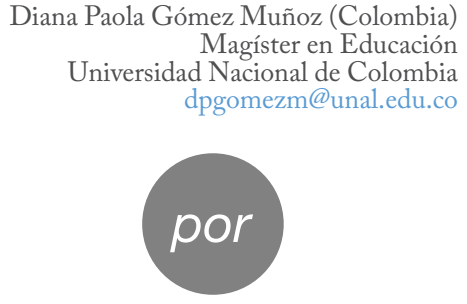

RESUMO

O presente artigo analisa a formação cidadã que oferecem as mães comunitárias de Bogotá a meninos e meninas da primeira infância. Para isto, se organizaram grupos focais com 27 mães comunitárias que fazem parte do programa Técnico en Atención Integral a la Primera Infancia do Servicio Nacional de Aprendizaje (SENA). Estabeleceram-se três categorias para a análise: participação, autonomia e pensamento cuidante. Realizou-se a visita ao Lar Comunitário Agrupado Domingo Savio da localidade Santafé, e neste exercício emergiram três novas categorias: cidadania nas mães comunitárias, juízo moral e resolução de conflitos, o qual permitiu ampliar a análise. $\mathrm{O}$ estudo arroja entre os seus resultados a importância de conceber aos meninos e as meninas da primeira infância como interlocutores válidos e compreendê-los como sujeitos políticos. Além do mais, se observou que as educadoras, desde a sua condição de género, aportam elementos para compreender a cidadania dos mais baixinhos e que a formação que oferece $o$ SENA tem tido um impacto positivo na resignificação da sua cidadania, o qual supõe uma afetação na orientação cidadã que promovem..

KEYWORDS: Citizenry, early childhood, community mothers, participation, autonomy, watching thought, conflict resolution, moral judgement.
PALAVRAS CHAVE: cidadania, primeira infância, mães comunitárias, participação, autonomia, pensamento cuidante, resolução de conflitos, juízo moral.

PARA CITAR ESTE ARTÍCULO/TO CITE THIS ARTICLE/PARA CITAR ESTE ARTIGO:

Rodríguez Huesa, A. A., Flórez Romero, R. y Gómez Muñoz, D. P. (2016). La formación en ciudadanía en escenarios de educación inicial: una experiencia con madres comunitarias. Panorama, 10(18), pp. 102-119. 


\section{IINTRODUCCIÓN}

Favorecer la formación en ciudadanía y participación desde la primera infancia resulta relevante, en cuanto se hace posible la construcción de entornos donde los niños y las niñas son escuchados y tenidos en cuenta por parte de los adultos, quienes han de reconocer que, desde el principio de sus vidas, los pequeños configuran un lenguaje en el que entretejen sus deseos, sus miedos y sus comprensiones sobre el mundo, teniendo el derecho a expresarlos. La sociedad, por tanto, debe ver la necesidad de construir ambientes para su desarrollo, que les permita crear y recrear su propia identidad de forma propositiva y participativa, al ser considerados por los adultos como interlocutores válidos y legítimos, en últimas, como sujetos políticos, en cuanto son sujetos de derecho.

Esta mirada hacia la infancia emerge a partir de la Convención sobre los Derechos del Niño de 1989, en la cual se establece el reconocimiento de los niños y las niñas como sujetos de derechos, y se les considera un grupo social fundamental ante el reconocimiento de nuevas emergencias sociales, con lo cual visibiliza su derecho a la participación en la sociedad.

Colombia ratificó esta Convención en la Constitución de 1991, de manera que el tema de la primera infancia ha suscitado cada vez mayor atención y prioridad, no obstante, es desde la última década cuando se han gestado mayores iniciativas por parte de diferentes instituciones y personas de todos los sectores sociales para plantear acciones más efectivas de intervención desde el Estado. Una de estas acciones dio lugar a la formalización del Documento Conpes Social de 2007 denominado Colombia por la primera infancia, que fue la primera política pública en toda su historia dirigida a este grupo poblacional (Torrado, 2009).

La política traza 10 lineamientos de acción, entre los cuales se encuentra el de Lineamiento técnico de participación y ejercicio de la ciudadanía en la primera infancia (Comisión Intersectorial para la Primera Infancia, 2012). Este Lineamiento es significativo, porque promueve la realización del conjunto de derechos de los niños y las niñas y se constituye en una de las orientaciones fundamentales de la política de atención integral que adelanta el país dentro de la Estrategia de atención integral a la primera infancia (Comisión Intersectorial para la Atención Integral a la Primera Infancia, 2014).

La investigación realizada centró su análisis en indagar sobre cómo se lleva a cabo la formación de ciudadanía en estos pequeños, para evidenciar la manera en que se estaba llevando a la práctica lo propuesto por este Lineamiento, y así se abordó un trabajo con madres comunitarias en busca de caracterizar su comprensión sobre la noción de ciudadanía y la relación de estos imaginarios con la enseñanza que imparten en este tema.

La primera infancia es entendida aquí como el grupo poblacional en el cual se encuentran los niños y las niñas de 0 a 6 años, periodo significativo en el proceso de formación del ser humano, porque allí se estructuran las bases fundamentales para el desarrollo cognitivo, emocional y social, que se consolidan y perfeccionan en las posteriores etapas del desarrollo (Ley 1098 de 2006).

\section{MARCO TEÓRICO}

\section{LA CIUDADANÍA}

Resultaba pertinente realizar un acercamiento al concepto de ciudadanía para entender cómo esta fue interpretada en este trabajo, y así se describen unos referentes teóricos. La modernidad concibió la ciudadanía como una noción ético-política a partir de la cual los seres humanos son reconocidos como sujetos que participan en igualdad de derechos y deberes en cuanto miembros de una sociedad.

El concepto de ciudadano actual es la reconfiguración de diferentes hechos históricos, como los dados en los siglos XVII y XVIII, en la época de la Revolución francesa, inglesa y americana y del nacimiento del capitalismo, como producto de las ideas liberales de la Ilustración. Uno de los grandes acontecimientos relacionados con esta noción fue el nacimiento de una nueva comunidad política: el Estado nacional moderno, como institución cuya responsabilidad es defender la vida, la integridad y la propiedad de sus miembros.

El Estado nacional moderno permitió la reconfiguración actual del concepto de ciudadanía; son ciudadanos que tienen la nacionalidad de un país, y esta es el

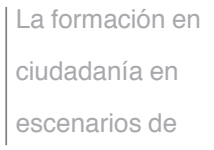

educación inicial: una experiencia con madres comunitarias 
estatuto legal por el que una persona pertenece a un Estado que haya sido reconocido como tal.

Arly Adriana

Rodríguez

Huesa I

Rita Flórez

Romero I

Diana Gómez

Muñoz |

Panorama I

pp. 102-119 |

Volumen 10 ।

Número 18।

Enero-junio I

2016 I

hacia el cuidado y la responsabilidad. El primero tenía viabilidad en el ámbito público, el segundo proyecto, en cambio, quedó confinado al ámbito privado, al campo doméstico, con lo cual se excluyó a la mujer de la esfera política (Pateman, 1986, citado por Mouffe, 1992, p. 7).

Si se atiende al devenir histórico sobre la ciudadanía de la mujer, se evidencia una exclusión en temas, como la participación democrática, razón por lo cual se entiende por qué muchas de las madres, en su condición de género, manifestaron no sentirse suficientemente reconocidas por el Estado, lo cual hace parte de una cultura de tipo patriarcal, que ha excluido e invisibilizado a la mujer.

Segundo, si bien el respectivo estudio no ahondó en las luchas que ha tenido que afrontar el feminismo por asentar una concepción de ciudadanía más incluyente para la mujer, fue necesario exponer unas consideraciones feministas, en cuanto brindaban elementos para entender qué aspectos naturales de la mujer confinados al ámbito privado desempeñan un papel fundamental en el ámbito público y dan elementos que deben tenerse en cuenta para formar en ciudadanía en la primera infancia.

En efecto, algunas autoras, como Mouffe (1992), Ruddick (1989) y Elshtain (2006), se han unido al pensamiento de Gilligan (1985), quien ha opuesto una "ética del cuidado" feminista a la "ética de la justicia" masculina y liberal. En contra de los valores individualistas liberales, defienden un conjunto de valores basados en la experiencia de las mujeres como mujeres; la experiencia de la maternidad y del cuidado que llevan a cabo en el ámbito privado de la familia.

Así señalan que existe una moralidad femenina como un modo diferente de razonamiento moral y una fuente de intuiciones morales auténticas y valiosas que deben ser visibilizadas. Ahora bien, si ser ciudadano exige poder participar en la vida social y pública, lo cual implica adoptar un punto de vista moral, bien puede afirmarse que este puede estar determinado por una condición de género y debe tener incidencias en el modo como se asuma el ser ciudadano por parte del sujeto (Palma Pizarro, 1997). De ser así podría afirmarse que, si existe tal determinación, esta tiene una afectación en el cómo se cultiva en los niños y las niñas el tema de la ciudadanía. Para ilustrar lo anterior, resulta pertinente señalar el siguiente estudio. 
Singer y De Haan (2008) analizaron algunos procesos de socialización en la primera infancia y afirman que una de las principales labores de las educadoras es asegurar que los niños se sientan seguros y valorados y que estén a gusto en el grupo y creen una "cultura de la ternura” (Singer y Hännikäinen, 2002, citado por Singer y Hann, 2008, p. 115), una cultura que se sustentaría en la ética del cuidado propuesta por Gilligan (1985). Este planteamiento surgió tras observar cómo las educadoras objeto de estudio asumían los procesos de resolución de conflictos de los pequeños (tema del cual se tratará más adelante). En efecto, distinguieron tres roles de las docentes:

\section{La protectora, quien vela para que todos los niños se sientan seguros y mantengan relaciones positivas. 2. El de autoridad, que se ocupa de los valores y las normas, establece reglas y acuerdos con los niños, cuando es necesario, interviene para recordárselos. 3. El de mediadora, quien ayuda a los niños a encontrar soluciones, conjuntas, restablece relaciones y reconcilia y, en general, fomenta el desarrollo de habilidades sociales (p. 115).}

\section{CIUDADANÍA EN LA PRIMERA INFANCIA}

La educación es fundamental para la formación ciudadana, sin embargo, dadas las crisis políticas, sociales y culturales del mundo actual y las múltiples transformaciones económicas producto del capitalismo y la globalización, la sociedad ha visto la necesidad de pensar y re-significar modelos educativos y nociones como ciudadanía e infancia. Esta última identificada ahora como un grupo social específico y no solo como una etapa de preparación para la vida adulta o para el futuro de la sociedad. Así, se ha empezado a hablar desde una concepción de la infancia que entiende al niño y a la niña como sujetos de derechos políticos, es decir que reconoce para la infancia la posibilidad de una ciudadanía constituida de hecho y no por alcanzar (Garzón, Pineda y Acosta, 2004, p. 8).

La pregunta emergente en esta parte de la indagación se planteó de la siguiente forma: ¿̇es posible hablar de ciudadanía en la primera infancia? Al respecto, Pineda (2004) afirma que los niños son seres sociales que experimentan ya desde muy pequeños todas las contradicciones de los contextos en que viven y que requieren, por tanto, empezar a clarificar desde el comienzo los conceptos e imágenes con que se tropiezan a diario.
Concebir al niño como un ser "puro", no contaminado y, en cierto modo, ajeno al contexto social en que se desenvuelve resulta muy parcial, pues desconoce no solo su curiosidad intelectual, sus capacidades de razonamiento, sus dotes perceptivas y la forma como sus incapacidades físicas y las limitaciones de su intelecto se compensan con una elevada capacidad de interacción social.

Las habilidades y competencias ciudadanas en los niños y las niñas requieren, por tanto, estrategias para una construcción continua, que deben desarrollarse en el mundo de la vida cotidiana (escuela, hogar, parque, barrio, ciudad...). Precisa de espacios que permitan la participación, la reflexión del infante y la puesta en práctica de su ciudadanía, en cuanto, como lo afirma Hart (2005), el niño y la niña:

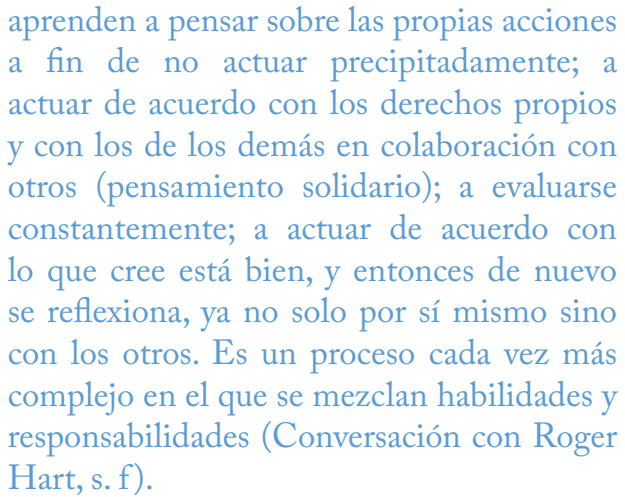

Estas consideraciones, que bien solo podrían pensarse para el mundo adulto, recuerdan las tres máximas presentadas por Kant (2006): pensar por sí mismo, pensar desde la perspectiva de los otros, pensar siempre de un modo consciente.

A partir de la anterior conceptualización, se pudo observar que para la formación en ciudadanía son importantes tres aspectos: la participación, la autonomía y el pensamiento cuidante. A continuación, se presenta la conceptualización de cada noción.

\section{Participación}

El estudio realizado se centró en el análisis del artículo 12 de la Convención sobre los Derechos del Niño, el cual se refiere al derecho que tiene toda niña y niño de expresar su opinión y a que esta se tenga en cuenta en todos los asuntos que los afectan. Zamora dice al respecto:

\section{La formación en ciudadanía en escenarios de} educación inicial: una experiencia con madres comunitarias 
Arly Adriana

Rodríguez

Huesa I

Rita Flórez

Romero I

Diana Gómez

Muñoz I

Hart (1993) define la participación como la capacidad para tomar decisiones que sean reconocidas por el entorno social y que afectan a la vida propia o a la vida en comunidad en la que uno vive. La participación infantil supone "colaborar, aportar y cooperar para el progreso común", así como generar en los niños, las niñas y los jóvenes confianza en sí mismos y un principio de iniciativa. Además, la participación infantil ubica a los niños y las niñas como sujetos sociales con la capacidad de expresar sus opiniones y decisiones en los asuntos que les competen directamente en la familia, la escuela y la sociedad en general. De igual forma, la participación infantil nunca debe concebirse como una simple participación de niños y jóvenes, sino como un hacer en permanente relación con los adultos (diálogo inter-generacional), y debe ser considerada como un proceso de aprendizaje mutuo tanto para los niños como para los adultos (Apud, s. f.).

La mayor parte de los documentos revisados sobre el tema de la participación infantil hacen referencia a la metáfora empleada por Hart (2001), conocida como la escalera de la participación infantil, para explicar cómo los adultos pueden presentar apoyo para la implicación de los niños y las niñas en sus procesos participativos, para evitar su manipulación y lograr modelos de participación y estructurar estrategias genuinas (Comisión Intersectorial para la Atención Integral a la Primera Infancia, 2012) (figura 1).
Volumen 10 I

Número 18।

Enero-junio I 2016 ।

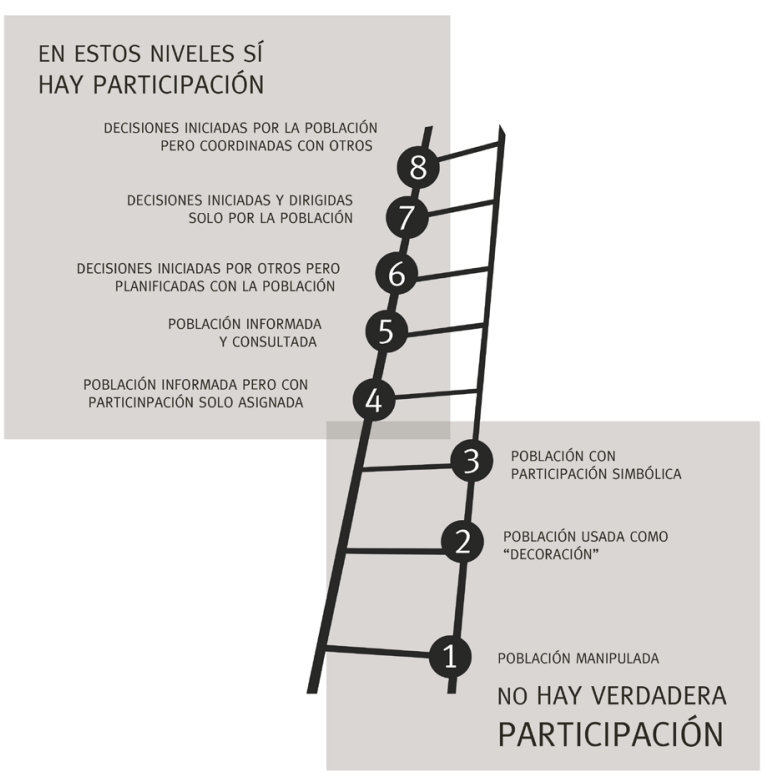

Figura 1. Escalera de la participación planteada por Hart (2001).

Hart (2001) afirma que en los primeros niveles no se da una participación real por parte de los niños y las niñas, por lo que destaca los escalones en los que esta sí tiene lugar, a saber:

Nivel 4. Asignados pero informados: este es el primer nivel de la participación genuina, aquí los niños y las niñas actúan de manera voluntaria en las propuestas de los adultos, porque estos han generado espacios de comunicación en los cuales se explican las actividades a los pequeños. En este caso, los niños y las niñas comprenden la intención de la actividad que se va a desarrollar, porque saben quién tomó las decisiones sobre su participación, y se conciben como personas significativas (no decorativas), y se ofrecen como voluntarios para el proyecto.

Nivel 5. Consultados e informados: un nivel superior de la participación lo constituyen aquellos proyectos en los cuales los niños trabajan como consultores de los adultos, porque las opiniones y propuestas de los niños y las niñas son tomadas en cuenta, de manera que los adultos ven la necesidad de construir estrategias que permitan hacer efectiva la escucha de los más pequeños.

Nivel 6. Iniciada por los adultos, decisiones compartidas con los niños y las niñas: si bien en este nivel los proyectos son iniciados por los adultos, la toma de decisiones se comparte con los niños y las niñas. De esta manera, se logra desarrollar un trabajo colaborativo y cooperativo, 
porque los adultos brindan la posibilidad para que los pequeños aporten desde su creatividad, imaginación, estilo y capacidad propositiva en la interacción con los adultos para el desarrollo del trabajo propuesto.

Nivel 7. Iniciada y dirigida por los niños: en el séptimo grado, los proyectos son iniciados y dirigidos por los niños y las niñas. Aquí se requiere de los adultos un cambio de actitud dirigido a reconocer y legitimar a los niños y las niñas como sujetos de derechos, creer en su capacidad de decidir y actuar desde sus propias iniciativas y estar dispuestos a brindar un acompañamiento en este proceso de crecimiento.

Nivel 8. Iniciada por los niños y las niñas, decisiones compartidas con los adultos: este último escalón se caracteriza porque los proyectos son encabezados por los niños y se comparten las decisiones con los adultos. Para que se alcance este nivel superior en el proceso de participación, se necesita que los adultos sean capaces de identificar las necesidades de los pequeños y posibilitar las condiciones pertinentes para que puedan desarrollar todo su potencial creativo.

Es preciso señalar que la participación social es un derecho humano esencial de toda persona y una sociedad puede considerarse democrática cuando todos sus ciudadanos y ciudadanas participan y son incluidos en ella y aseguar así el cumplimiento de sus derechos. Según el lugar que ocupan los niños y las niñas en su grupo social, dependerán los niveles y las formas de participación (Estrada, Madrid-Malo y Gil, 2000).

En resumen, la participación y el ejercicio de la ciudadanía está ligada a los procesos de desarrollo de los niños y las niñas en primera infancia y como tal a los patrones de crianza, a los procesos de educación inicial y a los imaginarios individuales y colectivos que sobre ellos y ellas se tengan. Considerar a los más pequeños como interlocutores válidos por parte de los adultos permitirá favorecer las capacidades y potencialidades necesarias para la participación de los niños y las niñas.

\section{Autonomía}

Uno de los logros más representativos de los niños hacia el final del primer año y gran parte del segundo es la autonomía, que emerge en esa transición del bebé al niño, porque deja de ser totalmente dependiente, para convertirse en un sujeto ejecutor. Es un momento de grandes conquistas: sus primeros pasos, la posibilidad de comunicarse, la capacidad de solucionar problemas, así como de la comunicación y los inicios del lenguaje, por lo que gradualmente empieza a vivir su libertad como persona autónoma.

Ahora bien, no es posible hablar de ciudadanos y ciudadanas sin su reconocimiento como seres autónomos y libres, luego las personas que acompañan al menor deben propender a favorecer la apertura de espacios de descubrimientos y logros, porque ello fortalece el camino para el desarrollo de su autonomía avanzando hacia la independencia. En efecto, los niños hacia los 2 años evidencian mayor interés en hacer las cosas por sí mismos y en ocasiones no desean recibir ayuda de los adultos que están a su alrededor. Este sentido de autonomía muestra la confianza que los pequeños tienen en sus propias capacidades, así, se arriesgan por sí mismos a tomar decisiones, aunque aún siguen requiriendo el apoyo de los adultos.

Los adultos que están con los pequeños tienen un papel fundamental en la conquista de este logro, pues deben permitir abrir espacios en los que el niño o la niña sienta confianza en sí mismo/a, para lograr la transformación de los "saberes" y "haceres" en "poder hacer", para lo cual requieren tiempo y libertad de actuar. Este poder hacer se entrelaza con afectos, ideas y sentimientos, por lo cual sienten la necesidad de explorar e investigar el ambiente físico, de tomar decisiones y de hacer cosas por sí mismos. Estos cambios son el inicio del desarrollo de su independencia, de la construcción de su autoestima y la definición de su personalidad.

Este proceso de desarrollo y construcción de seres autónomos es fundamental para hacer viable que el niño y la niña puedan tomar decisiones y declarar puntos de vista ante una situación determinada. En efecto, la autonomía entendida como una tendencia biológica se encuentra estrechamente relacionada con la autoestima y con la felicidad, de forma que una persona autónoma tiene conciencia de la posibilidad de idear y soñar proyectos con la confianza de que puede realizarlos.

Trasladar esta reflexión al ámbito de la primera infancia significa que los agentes educadores deben inculcar en los pequeños la valoración de su propia vida, el (n)

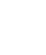


Arly Adriana

Rodríguez

Huesal

Rita Flórez

Romero I

Diana Gómez

Muñoz I

Panorama I

pp. 102-119|

Volumen 101

Número 18।

Enero-junio I

2016 I

Esta consideración de sí mismo, del otro y de lo otro, característica del pensamiento cuidante, es importante para el cultivo de la ciudadanía y, por ende, de la democracia. Se quiso establecer una relación entre las consideraciones de Lipman sobre el pensamiento cuidante y

reconocimiento como personas valiosas y construir así las bases para que más adelante sueñen con sus propios proyectos de vida y enseñar que sus vidas tienen sentido y fines, entre ellos, procurar ser feliz.

\section{El pensamiento cuidante}

Lipman (1998) desarrolló la dimensión del pensamiento cuidante en cuanto el razonamiento y el juicio moral cobra sentido si hacen referencia a la preocupación por uno mismo, por los demás y por el mundo. Este autor vincula su reflexión con la conceptualización heideggeriana del cuidado o cura, al definirlo como una manera auténtica de estar o de ser en el mundo y de relacionarse con el entorno y con los demás (Lago, 2006). El aporte de Lipman fue considerar que el cuidado, además de ser una actividad emotiva o afectiva, es un acto cognitivo y reflexivo, puesto que implica la realización de operaciones cognitivas del tipo buscar alternativas, descubrir o inventar relaciones, establecer conexiones entre conexiones y contrastar diferencias. Acciones que se realizan cuando cada quien indaga sobre su relación con el mundo y con los demás.

Lipman (1998) afirma que, cuando se está pensando cuidadosamente, se presta atención a aquello por lo que nos preocupamos, por ello le otorga un carácter valorativo, en cuanto emite un juicio sobre la persona, sus acciones y conductas y sobre el mundo que lo rodea, por tanto, es un pensamiento comprometido con la persona y con el entorno. Esta preocupación por el otro o lo otro supone la preocupación por uno mismo, es decir, supone el cuidado de sí. De esta manera, el pensamiento cuidante resulta fundamental para la construcción de la ciudadanía, porque tiene un carácter social (aquí se separa de las consideraciones de Heiddeger para una mirada más foucaultiana), pues se trata de tener conciencia de uno mismo a través de los demás, en la interacción con los otros y con el entorno, se trata de una conciencia activa de la persona en cuanto se "preocupa por" y procura cuidarlo, transformarlo, es decir que se proyecta en los otros y en lo otro (Lago, 2006).

los planteamientos de Nusbaum (2010), para entender cómo las implicaciones de este pensamiento estarían vinculadas a las acciones que deben corresponder a un ciudadano.

La autora afirma que para la formación de la ciudadanía mundial es preciso el desarrollo de tres capacidades: la capacidad de autoexamen (conciencia de uno mismo), la capacidad de verse a sí mismo como humano que está en interdependencia con los otros (conciencia de uno mismo a través de los demás) y la capacidad del cultivo de la imaginación narrativa (preocuparse por).

La capacidad de autoexamen propende al ejercicio autorreflexivo y la construcción del pensamiento crítico sobre el mundo de la vida. La segunda capacidad implica la posibilidad de verse a sí mismo, que está en relación con los otros, que conlleva trascender las fronteras culturales en cuanto está dirigido a una ética del cuidado del mundo, de lo otro. La tercera capacidad es la imaginación narrativa que implica el sentimiento de empatía, de ponerse en lugar de los otros para valorar el significado de sus sentimientos, sus deseos, proyectos y logros.

Dado lo anterior, fue posible afirmar que el pensamiento cuidante es fundamental para la formación de la ciudadanía, porque conlleva las tres consideraciones de las que habla Nussbaum (2010), pues este pensamiento pone al sujeto como agente reflexivo de sí, reflexión que le permite: la valoración de lo que valoran los otros, pensamiento respetuoso, pensar lo que se valora como bello, pensamiento apreciativo, pensar lo que se considera virtuoso, pensamiento admirativo, pensar lo que es sintiente, pensamiento considerado, pensar lo que se considera debe ser apoyado, pensamiento animante, pensar lo que es el sufrimiento, pensamiento compasivo (Lipman, 1991).

\section{Metodología}

\section{TIPO DE ESTUDIO}

El análisis de la investigación corresponde a estudios de caso múltiple, se utilizó un enfoque cualitativo de tipo descriptivo, interpretativo, enfocado en la generación de un conocimiento comprensivo sobre la concepción de ciudadanía de las madres comunitarias y su relación con la formación ciudadana en la primera infancia, sustentado en una perspectiva hermenéutica. 


\section{INSTRUMENTOS Y PROCEDIMIENTO}

Se siguió la técnica del grupo focal, el cual estuvo conformado por 27 madres comunitarias de la localidad de Santafé; los debates en ese grupo de discusión estuvieron guiados por preguntas previamente diseñadas y se realizaron algunas entrevistas, con el fin de explorar la formación en ciudadanía en la primera infancia, a través de las categorías de análisis: participación, autonomía y pensamiento cuidante.

La investigación se desarrolló en tres momentos: en primer lugar, se realizaron los grupos focales con las madres comunitarias que estaban dentro del proceso de formación en Atención Integral a la Primera Infancia. En segundo lugar, se visitó el Hogar Comunitario Integrado Domingo Savio, con el fin de observar e identificar situaciones relacionadas con las preguntas de estudio: ¿cómo forman en ciudadanía las madres comunitarias?, ¿qué sentido tiene?, ¿es posible hablar de ciudadanía en la primera infancia? Esta indagación fue guiada por el respectivo diario de campo. En el proceso de diálogo y observación, emergieron nuevas categorías de análisis que permitieron ampliar la mirada sobre la formación ciudadana en los niños y las niñas. Y en tercer lugar, se realizó un análisis de los conceptos emergentes: ciudadanía en las madres comunitarias, resolución de conflictos y juicio moral.

Por otra parte, uno de los insumos importantes en la investigación fue el proyecto de grado para recibir la titulación como Técnico en Atención Integral a la Primera Infancia del Servicio Nacional de Aprendizaje (SENA), realizado por cuatro madres comunitarias del Hogar Comunitario Agrupado Domingo Savio en 2012, porque en él se consignaban las actividades encaminadas a la formación ciudadana en el hogar comunitario de estudio y se constituye así en una fuente importante de información sobre las prácticas pedagógicas adelantadas por las docentes.

\section{PARTICIPANTES}

El grupo de estudio lo conformaron 27 madres comunitarias que hacen parte del programa Técnico en Atención Integral a la Primera Infancia del SENA; cuatro de estas madres ya habían culminado su proceso de formación en 2012. Las agentes educativas tienen sus hogares en la localidad de Santafé. La institución de estudio fue el Hogar Comunitario Agrupado Domingo Savio.

\section{CATEGORIZACIÓN}

La organización de la información recopilada se realizó a partir de tres categorías de análisis, que surgieron de la contextualización teórica sobre ciudadanía, y a partir de estas unidades analíticas se interpretaron los relatos, se comprendieron aspectos dados en la observación y se revisó el proyecto de grado del programa Técnico en Atención Integral a la Primera Infancia del SENA, lo cual se convirtio en nociones significativas para la configuración de los resultados obtenidos. En el transcurso de la investigación, surgieron otras tres categorías que ampliaron el análisis del estudio.

\section{RESULTADOS}

A continuación, se describen las actividades realizadas por las madres, que respondían a los elementos de análisis escogidos.

\section{ACTIVIDADES OUE DAN CUENTA DE LA PARTICIPACIÓN}

Para indagar sobre esta categoría, se les preguntó a las madres comunitarias por las actividades que desarrollaban para esta formación. Los resultados evidenciaron, por un lado, el planteamiento de actividades en las que el nivel de participación de los niños y las niñas no era del todo genuina, según la descripción de los grados de participación propuestos por Hart (1993). A continuación, se mencionan algunas de estas actividades:

Nivel 2. Se organizó una actividad donde los niños y las niñas tuvieron que salir a bailar, en el día de la familia y tenían que representar una canción, se disfrazaron de los personajes. Ellos, al principio, tenían susto, pero los motivamos dándoles un aplauso y los chiquitines empezaron a bailar; todo salió bien y a los papás les gustó (madre comunitaria 4).

Nivel 3. En el hogar, se trabaja la participación en las elecciones de personero o se escoge a un niño o una niña que represente el salón o el jardín (madre comunitaria 2).

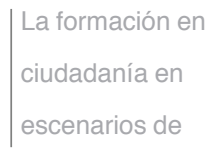

educación inicial: una experiencia con madres comunitarias 
Arly Adriana

Rodríguez

Huesa I

Rita Flórez

Romero I

Diana Gómez

Muñoz I

Panorama I

pp. 102-119|

Volumen 101

Número 18 ।

Enero-junio I

2016 I

mera, el guarda de seguridad, las madres y los padres y a ellos mismos en calidad de pacientes (Grupo Jardín A2, Hogar Agrupado Comunitario Domingo Savio, con la colaboración activa de los niños y las niñas usuarios del 110

En estas actividades, se evidencia cierta pasividad por parte de los niños y las niñas, si bien se integraron en ellas, no se abrieron mayores espacios para que los pequeños se expresaran más y compartieran sus opiniones y percepciones. Otras actividades, en cambio, propiciaban un mayor protagonismo de los pequeños y les brindaban la oportunidad de tomar decisiones, sin embargo, la participación no era del todo efectiva, por ejemplo:

Nivel 4. La actividad fue sobre el tacto gustativo, les dimos a probar un limón y después miel; algunos niños hacían muecas, pero se devolvían para que les diéramos más limón, como son niños de 1 año a 1 año y medio, se sonreían al ver las reacciones de los otros y luego salían corriendo [...] así participaron todos. En esta actividad, los pequeñitos decidían qué querían, si miel o limón, miraban y comentaban las reacciones de unos y otros (madre comunitaria 8).

Sumariamente, se encontraron actividades que sí posibilitaban una participación genuina por parte de los pequeños y que propendían, además, al desarrollo de pensamiento de tipo crítico, cuidante y solidario. A continuación, se menciona una de ellas:

Nivel 8. Con los niños y las niñas de este nivel se trabajó el tema de los hospitales y centros de salud de la localidad... se realizó un mural donde ellos expresaron lo que les gustaba y lo que no les gustaba de estos lugares. En el mural de lo que no les gustaba, mostraron un hospital feo, sucio, con paredes en mal estado. Adicionalmente, prepararon una representación con la ronda $L a$ canción de Pinocho, para hacer alusión al viejo hospital donde se atiende mal a Pinocho, con lo cual manifestaban la mala atención que ellos reciben cuando van al médico. Los pequeños representaron el hospital que querían y deseaban. Previamente, se visitó el Hospital Jorge Eliécer Gaitán, y la UBA [Unidad Básica de Atención] de Lourdes; allí tuvieron contacto con los distintos profesionales, quienes les contaron los servicios a que tienen derecho los niños y las niñas. Posteriormente, en el hogar comunitario, desarrollaron un juego de roles donde representaron los papeles del médico, la enferHogar Niños Soñadores).
Las madres comunitarias, en su mayoría, consideraron durante los grupos focales que el arte y el juego son medios fundamentales para fortalecer la autonomía de los pequeños a su cargo, porque en estos ámbitos los niños y las niñas tienen la oportunidad de explorar, indagar, preguntar, ser propositivos, de equivocarse y corregir, de tomar decisiones, desde los más pequeños hasta los más grandes.

Sin embargo, en la visita al hogar comunitario se logró entrever otra realidad: que la formación en autonomía la realizan las educadoras más desde la construcción del pensamiento normativo. Las normas por seguir por parte de los niños y las niñas en el hogar comunitario se socializan al comenzar el año, pero constantemente están siendo reforzadas en la interacción que tienen con los pequeños.

Si bien la autonomía no se reduce al seguimiento de lo normativo, pues ello implica una capacidad de discernimiento por parte de la persona para que realmente esta tenga lugar, se logró observar que desde la asimilación de la norma las madres comunitarias logran que los niños y las niñas tengan claro qué hacer, cómo hacerlo y se sienten seguros al poder colaborar de las labores encomendadas por ellas. Si bien las educadoras están muy pendientes de los pequeños, dejan que sean ellos quienes solucionen sus problemas (comer solos, alcanzar una silla, organizar la loza, ordenar los juguetes, dejar las cosas personales en su sitio, desde los pequeños de 1 año hasta los más grandes de 5 años), y fortalecer así el ejercicio de la autonomía.

ACTIVIDADES QUE DAN CUENTA DEL PENSAMIENTO CUIDANTE

Las madres comunitarias en su proceso de formación en el programa Técnico en Atención Integral a la Primera Infancia del SENA reciben un aprendizaje orientado hacia la promoción de la salud, la nutrición, las prácticas de vida saludable y la prevención de enfermedades de niños y niñas hasta los 6 años, de acuerdo con lineamientos de salud pública, por esta razón varios de los testimonios orientados a saber cómo enseñaban sobre el tema del cuidado referenciaron este aspecto. Sumariamente, las madres trabajan en la promoción de la 
interacción idónea consigo mismas, con los demás y con la naturaleza, como se evidencia en las siguientes actividades.

"Sobre el cuidado del otro". En la visita al hogar comunitario, se pudo observar cómo las madres comunitarias procuraban que los niños y las niñas más grandes estuvieran atentos de los más pequeños, así, cuando se hizo la actividad del baile, todos seguían la siguiente instrucción por parte de la educadora: "Los grandes tomen como pareja un bebé”, o decían en el momento del juego: "Dejen que los más pequeños cojan primero su juguete". Las indicaciones reiteradas hacia el cuidado de los pequeños han llevado a que los más grandes moderen sus comportamientos para no lastimarlos y a tener iniciativas propias para protegerlos. Así un pequeño de 2 años le dijo a la profesora: "Mira que otro bebé se quiere salir”, señalando a un gateador y cerrando el corral.

Respecto del cuidado de lo otro, muchas de las actividades realizadas por las madres comunitarias están orientadas hacia el cuidado y la protección de la naturaleza y de sus entornos inmediatos, además idean actividades relacionadas con el conocimiento de las calles y normas de tránsito, lo cual es un reto, dado que, como lo plantea Tonucci (1991), las ciudades se han convertido en lugares donde circulan muchos carros, lo cual dificulta el derecho que tienen los pequeños de usar los espacios de la ciudad de una manera más libre y segura. En efecto, la mayoría de los espacios urbanos son hostiles para cualquier pequeño.

\section{CATEGORÍAS EMERGENTES}

\section{Ciudadanía en las madres comunitarias}

Uno de los intereses de este estudio estuvo centrado en indagar sobre la concepción que tienen las madres comunitarias de la ciudadanía y ver su incidencia con la formación que brindan a los pequeños en este aspecto. En los referentes teóricos, se afirmó que existe una condición de género que afecta el cómo asuman las madres comunitarias su propia ciudadanía, razón por la cual, por un lado, se preguntó qué pensaban de ella, y por otro, con el fin de profundizar la reflexión, se trató el tema de sus derechos. $\mathrm{Al}$ respecto, se encontraron resultados interesantes, como se describe a continuación.
En el cuadro 1 se registra en orden descendente las respuestas que dieron las madres comunitarias cuando se indagó sobre el qué las identificaba como ciudadanas.

Cuadro 1. Respuestas de las participantes acerca de qué las identificaba como ciudadanas

\section{Pertenecer a una comunidad, cultura, país}

Tener un nombre

Tener nacionalidad

Tener documento de identidad

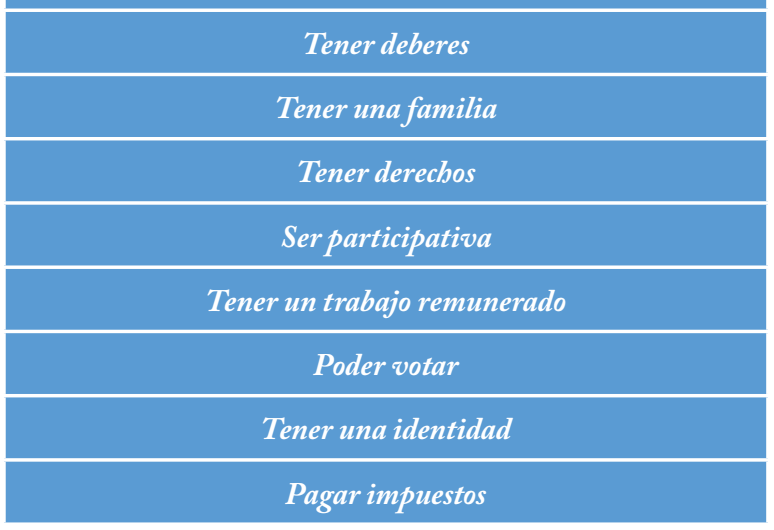

Ahora bien, uno de los problemas para el no reconocimiento práctico del pleno derecho de la ciudadanía en sus dimensiones jurídica, cultural, social, económica y política por parte de la mayoría de las personas es el enfocar el ser ciudadano solo hacia el tener un nombre, un documento de identidad o pertenecer a una patria. Las respuestas de las madres comunitarias estuvieron inclinadas hacia estos aspectos, pero otros, como el derecho al voto, que resulta ser un elemento fundamental para el ejercicio ciudadano porque representa una participación política genuina, no fue suficientemente tomado en cuenta.

Esto resultaba significativo para comprender la formación que ellas brindan a los pequeños, porque, si no han potencializado suficientemente el ser ciudadano, pues como afirma Cortina (1997), la ciudadanía va más allá de estos elementos al ser un valor en el que toda persona debe formarse para robustecer la convivencia política, entonces cómo logran formar en una ciudadanía real, puesto que no se puede dar de aquello que se carece.

Por otra parte, cuando se indagó sobre cómo viven la ciudadanía y cómo la proyectan, se encontró que su hacer como madres comunitarias, en algunos casos, no $\mid \begin{aligned} & \text { La formación en } \\ & \text { ciudadanía en } \\ & \text { escenarios de }\end{aligned}$

educación inicial:

una experiencia

con madres

comunitarias 
Arly Adriana

Rodríguez

Huesa I

Rita Flórez

Romero I

Diana Gómez

Muñoz I dista del rol privado como madre, esposa y ama de casa, lo cual tiene una afectación sobre su propia autonomía. En el trabajo realizado con las educadoras, resultó notorio cierto temor por expresar sus opiniones o defenderlas. Algunas madres manifestaron que sus maridos no las apoyaban con el proceso de formación que habían iniciado en el SENA, pues les resultaba difícil admitir que sus esposas estuvieran mejor preparadas que ellos, por eso unas se vieron obligadas a retirarse. De manera que fue necesario indagar sobre los derechos que ellas consideran les son reconocidos (figuras 2 y 3 ).

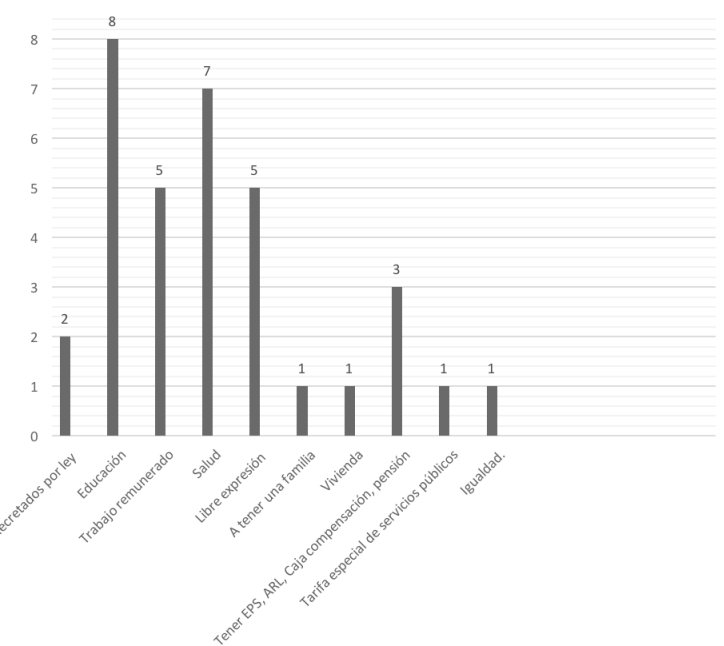

Figura 2. Derechos reconocidos por las madres comunitarias.

Nota: La educación hace referencia a la educación primaria y media.

Panorama pp. 102-119 Volumen 10 I Número 18 Enero-junio I 2016 |

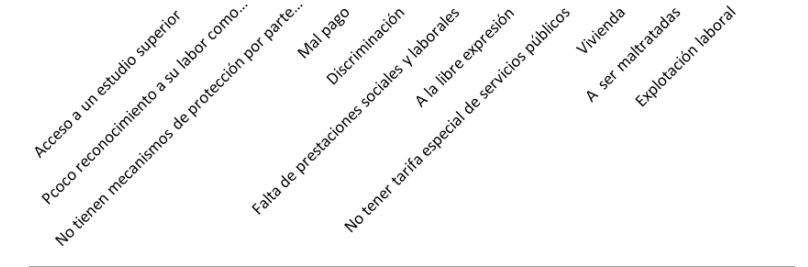

Figura 3. Derechos de las madres comunitarias vulnerados.
Como se puede observar en las figuras 2 y 3, las madres comunitarias en alto porcentaje manifestaron el no reconocimiento de derechos fundamentales para tener una vida mejor. Esta situación interfiere para un mejor ambiente laboral, muchas manifestaron que se debería reconocer más su labor, otras afirmaron que si existiera otra oferta laboral no dudarían en abandonar su trabajo y vincularse en otro (principalmente las madres más jóvenes hacieron esta afirmación).

Otras manifestaron que llegaron a realizar tal labor porque la vieron viable en su momento, movidas por una necesidad de acceder a algún recurso económico, mas no por una convicción personal de querer trabajar con niños y niñas. Estas y otras consideraciones deben despertar una alarma a la sociedad toda, porque la carga emocional que genera lo descrito, como el estrés, la frustración, la inconformidad, se ha de manifestar en el trabajo que hacen con los niños y las niñas. Luego cabe la pregunta ¿cómo logran hablar de derechos a los pequeños cuando no se reconocen suficientemente como sujetos de derechos, pues estos no son reconocidos por los estamentos pertinentes?

Por otra parte, cuando se indagó sobre cómo creían que formaban en ciudadanía, sus comentarios estuvieron orientados hacia el ámbito de la ética del cuidado más que hacia una ética de la justicia (figura 4).

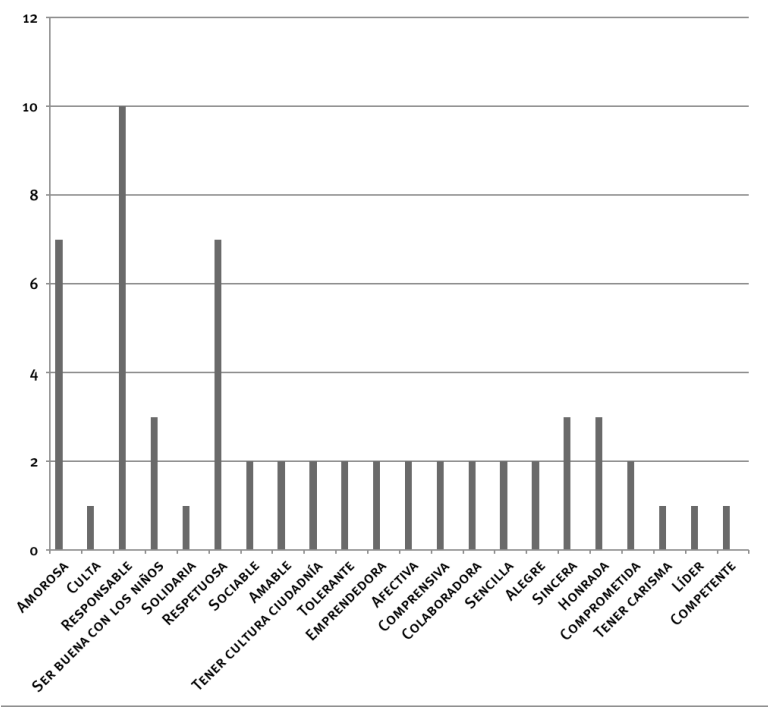

Figura 4. Indicadores de ciudadanía de las madres comunitarias desde la ética del cuidado. 
Según estos registros, bien puede señalarse que en la medida en que los agentes educativos resignifiquen la concepción que tienen sobre su propia ciudadanía tendrán mayores elementos para formar a los niños y las niñas que tienen a su cuidado. Así como no hacerlo tendrá una incidencia negativa en este proceso. En efecto, si una persona que desempeña el papel de agente educativo no se siente plenamente participativo y reconocido, cómo va a formar en participación, o si manifiesta el desconocimiento de sus propios derechos y considera que algunos son vulnerados pero no reclama su protección y cumplimiento, cómo va a formar en autonomía o en la consideración de sujeto político.

Es importante destacar que los registros dieron cuenta de que las madres forman en ciudadanía atendiendo a unas características relacionadas con su condición de género, lo cual haría pensar que forman desde una ética del cuidado.

Resultaría pertinente, por tanto, atender a estos elementos que brindan las docentes como mujeres, porque recrean la idea de cultivar formas de socialización desde una cultura de la ternura. Esta investigación trata de señalar que, precisamente, la afectividad y un mayor cultivo en la sensibilidad ha sido un elemento que no se ha tenido del todo en cuenta a la hora de plantear cómo formar en ciudadanía al caer en discursos meramente racionales, que atienden a una lógica de la justicia liberal.

\section{Resolución de conflictos}

A esta categoría se llega, porque cuando se indagó sobre cómo forman en ciudadanía las madres comunitarias manifestaron que uno de los inconvenientes que se presentan en su quehacer está relacionado con la dificultad para resolver problemas entre ellas mismas, lo cual afecta a los niños y las niñas, puesto que ellas no siempre trabajan de manera colaborativa y se presentan malos entendidos. Así, se examinó el tipo de conflictos que se presentan, bajo la consideración de que una de las virtudes de la vida en comunidad es la capacidad que deben tener los ciudadanos para establecer acuerdos, con lo cual se favorece la igualdad, entendida como el reconocimiento del otro desde su diferencia y, por tanto, la convivencia pacífica. De manera que no saber cómo llegar a acuerdos afecta significativamente la formación ciudadana.
Este análisis se llevó a acabo atendiendo a las siguientes relaciones y conflictos que se presentan entre ellas:

1. Madres comunitarias: entre las principales causas de situaciones conflictivas entre las madres comunitarias, ellas destacaron:

A. Falta mayor comunicación y buen entendimiento entre ellas. "No se aceptan los errores, algunas tienen actitudes conflictivas, porque no se respetan las ideas de las compañeras" (madre comunitaria 23).

B. Falta de una buena imagen de autoridad. "En ocasiones, se presentan diferentes desacuerdos con las líderes de las asociaciones, porque no saben escuchar, son intolerantes e impositivas" (madre comunitaria 13).

C. Desmotivación por el trabajo. "Se presentan permisos injustificados, lo cual afecta la labor con los niños porque el niño y la niña no identifica la imagen de la profe" (madre comunitaria 8).

D. Falta de solidaridad y reconocimiento por parte de las demás. "Algunas trabajan en beneficio propio y no en el de las demás" (madre comunitaria 14).

E. Se presenta falta de cumplimiento y de compromiso con las labores asignadas por parte de algunas compañeras.

Ahora bien, suele suceder que las docentes enseñan resolución de conflictos, pero pocas veces reflexionan sobre cómo manejan ellas mismas el conflicto en sus vidas. Las profesoras dan una estructura que capacita a los niños que no siempre tiene resultados eficaces, por lo que una buena alternativa sería que ante los conflictos los agentes educativos reflexionen y dialoguen con los niños y las niñas acerca de las situaciones problémicas que se presentan en sus propias vidas. Los profesores, padres y en general la comunidad deben encarar esta lucha de aprender a solucionar conflictos (Hart, 2005), para una enseñanza más efectiva, en busca de hacer acercamientos más de tipo vivencial que discursivos.

2. Madres comunitarias, padres de familia: las madres comunitarias afirmaron que los conflictos que se presentan con los padres de familia tienen como causas:

A. Falta de comunicación padres e hijos.

B. Falta de pautas de crianza.

C. Falta de tiempo de calidad con los hijos.

D. Familias agresivas o padres consumidores de sustancias psicoactivas.

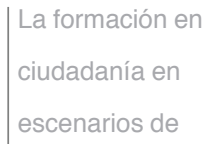

educación inicial: una experiencia con madres comunitarias 
Las madres comunitarias hicieron referencia ampliamente a la poca responsabilidad que asumen algunos padres de familia para la formación de sus hijos, además de ser intolerantes e irrespetuosos con ellas. Si bien en

Arly Adriana

Rodríguez

Huesa I

Rita Flórez

Romero I

Diana Gómez

Muñoz I

3. Conflictos entre la comunidad infantil: cuando se buscó información sobre este aspecto, llamó la atención que las madres comunitarias solo mencionaran las siguientes causas de los problemas que se presentan entre los infantes:

A. Egoísmo: "Suelen pelear mucho por los juguetes, no quieren compartirlos" (madre comunitaria 6).

B. Racismo: "Al jardín entró un niño negrito y al principio no querían jugar con él, fue preciso hablar con los niños para que lo aceptaran" (madre comunitaria 8).

C. Discriminación: "Se manifiesta cuando algunos de los niños y las niñas tienen alguna discapacidad, asî que trabajamos con ellos sobre la importancia de aceptar y respetar a los compañeros que presentan esta situación" (madre comunitaria 21).

Es preciso anotar que la presencia de conflictos en los procesos de socialización de la primera infancia pueden ser también momentos de grandes aprendizajes. De manera que no haber manifestado más conflictos llevó a la interpretación de que estos procesos no son observados suficientemente, con lo cual las madres comunitarias no alcanzan a visibilizar que los niños tienen la capacidad de llegar a soluciones y bosquejar acuerdos, como lo señalan Singer y De Haan (2008), luego no se fortalece esta habilidad.

Panorama

pp. 102-119

Volumen 10 I

Número 18

Enero-junio I

2016

En efecto, la forma como proceden los niños ante un conflicto resulta fundamental para la formación ciudadana y, por ello, debe fomentarse aún más esta actitud, la cual dista de la de los adultos que siempre están buscando tomar venganza y desquitarse. Así que resulta necesario que los adultos atiendan más los procesos de socialización de los infantes, para encontrar elementos que aporten a la ciudadanía desde las lógicas de los más pequeños.

\section{El juicio moral}

El desarrollo moral es uno de los procesos más importantes en la infancia, en el cual desempeña un papel importante el adulto al aprobar y desaprobar los comportamientos del pequeño, quien es la fuente y el paradigma para la construcción de sus valores. Sobre los sentimientos que experimentan y los efectos de sus conductas, los niños construyen paso a paso el andamiaje familiar y social en el cual están insertos (Winnicott, 1975).

Las madres comunitarias orientan esta formación hacia aprendizajes de socialización, conocimiento de las normas y los valores de la cultura y la familia, con lo cual van apoyando la configuración de criterios morales en los niños y las niñas. De forma que a partir de unos criterios que fijan límites de conducta las madres comunitarias forman en juicios morales. En cada una de las actividades descritas, se evidenció este proceso, y la manera como participen las niñas y los niños en cada uno de los ámbitos en los que se desenvuelven permitirá fomentar el desarrollo de su capacidad de crítica, de valoración ética, moral y cívica.

Las madres comunitarias consideran que inculcar en los niños y las niñas el respeto por los valores morales es fundamental por cuanto el ser humano se reconoce como constructor de estos. Pertenecer al programa Técnico en Atención Integral a la Primera Infancia del SENA les ha permitido ver que este saber es más significativo para los niños y las niñas desde un aprendizaje experiencial y no desde el estar repitiendo en el aula qué son los valores. Este conocimiento se aprende en la gramática de lo cotidiano, en medio de las relaciones que se entretejen entre ellos y con las madres comunitarias, por lo que resulta peligroso caer en un dogmatismo que pretende inculcar un conjunto de valores prefijados; este conocimiento debe estar dirigido a desarrollar en los pequeños la capacidad de buen juicio en cuestiones morales, lo cual implica estimular el buen razonamiento moral y la capacidad de análisis de las situaciones en las que los niños y las niñas están inmersos, ayudarlos a ampliar sentimientos y emociones acordes con las situaciones que viven y desarrollar una especial sensibilidad para colocarse en las perspectivas de otros cuando estos se encuentran en circunstancias de dilema o conflicto. 
La constitución del menor como sujeto moral no se define desde el punto de vista de responder a unas exigencias que le plantea la sociedad (padres, maestros y otras autoridades), es decir, de una moralidad externa, sino que se define principalmente respecto de sus posibilidades de una moral autónoma, construida desde su propio ejercicio reflexivo y comprensión ética del mundo del cual forma parte.

\section{DISCUSIÓN}

En este apartado, se realizarán algunas observaciones sobre la importancia de formar en resolución de conflicto y juicio moral para favorecer el proceso de formación ciudadana. Ha de señalarse que resulta normal que surjan conflictos entre niños, como cuando comparten el juego o se molestan unos a otros, porque cada uno quiere o descubre algo distinto y, a veces, se enfadan mucho con el otro.

Básicamente, los conflictos entre niños de 2 y 3 años se producen por los siguientes factores (Singer y De Haan, 2008, pp. 95-96):

1. Querer que lo dejen en paz.

2. La insistencia de querer participar en algo.

3. Conflictos relacionados con ideas sobre el juego.

Cada una de estas situaciones son espacios valiosos para la formación ciudadana, en efecto, los conflictos que surgen del deseo de ser dejado en paz brindan al niño y la niña la oportunidad de interiorizar algunos principios morales básicos: deberían dejar al otro en paz si así él lo desea. Porque los niños deben aprender a respetar tanto el espacio psicológico como el territorio físico de los demás y aprender a determinar hasta dónde va su libertad e inicia la libertad del otro.

La insistencia de participar en algo, afirman Singer y De Haan (2008), implica más que el dominio de las reglas sociales el hecho de saber utilizar las habilidades sociales adecuadas, puesto que el niño o la niña tienen que encontrar la mejor manera para poder participar; más o menos la mitad de los intentos de participar en el juego de otros fracasa y raramente surten efecto en el primer intento, $y$ como norma se observa que solo se logra participar tras uno o dos fracasos. Así que, para aumentar las posibilidades participación, el pequeño tendrá que hacer intentos de persuasión, como imitar el juego, ofrecer un objeto que encaje en el juego, sonreír o abrazar al otro.

De acuerdo con lo anterior, se tiene que la resolución de conflictos brinda la oportunidad para formar en ciudadanía, porque a partir de estas situaciones conflictivas se pueden enseñar a los niños y a las niñas principios fundamentales para la vida democrática: aprender a manejar su libertad, reconocer normas de convivencia, tener en cuenta las opiniones de los demás, manejar intereses opuestos y procurar participar en la comunidad.

Como afirman Singer y De Haan (2008), este aprendizaje empieza con los niños de 1 a 3 años; cuando un niño convive con catorce niños más de esa edad, la posibilidad de que surja un conflicto de intereses, necesidades u opiniones es constante, sea por ver quién se queda con el camión de bomberos, sea por quién puede participar en el juego. Además, en un grupo de niños de esta edad, algunas cosas son escasas, luego aprenden la importancia de compartir con otros. Los caminadores aprenden también a decidir sobre cuestiones tan esenciales como si admitir o excluir a otro en el juego. Aprenden a tener en cuenta a los demás, cuando se les propone jugar por turnos, a ser capaces de mirar desde la perspectiva de los otros, a manejar las diferencias y a conectar con los demás.

Así es como, en este trabajo, se entendió por desarrollo moral la vía que recorre el niño desde la adquisición de la moral heterónoma (imposiciones de afuera) a la autónoma (convicción personal), que le permite tomar conciencia de sí y del otro, decidir sus actos y valorar su influencia en la colectividad.

Al ser el desarrollo moral determinante para la formación ética y política del niño y la niña, es importante conocer y comprender los espacios de socialización que configuran entre pares, teniendo en cuenta, además, las circunstancias en que cada uno se desenvuelve, su origen, sus particularidades, su identidad familiar y social, para inculcar patrones que hagan al niño y a la niña seres capaces de construir colectivamente las normas, al igual que racionalizarlas y justificarlas. $\mathrm{El}$ ámbito por excelencia para formar personas autónomas es la vida cotidiana (Estrada, Madrid-Malo y Gil 2000).

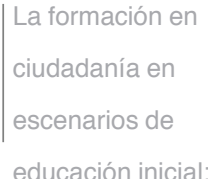

educación inicial: una experiencia con madres comunitarias
I Panorama |pp. 102-119 I Volumen 10 I Número 18 Enero-junio 


\section{CONCLUSIONES}

Entre los aspectos más representativos de la política por la primera infancia en Colombia, está dar prioridad a la construcción de estrategias para garantizar el desarrollo integral de niños y niñas. Dentro de este ordenamiento jurídico, se destaca su apuesta por favorecer la participación y el ejercicio de la ciudadanía, para que desde sus voces como ciudadanos activos sean conscientes y exijan el cumplimiento de sus derechos. Lo cual resulta significativo, porque, como afirma Baratta (citado por Acosta y Pineda, 2007), la cuestión de la democracia tiene que ver con la posibilidad de admitir la ciudadanía de los niños y las niñas.

Lamentablemente, las necesidades y potencialidades de la infancia no son de un modo suficiente conocidas y reconocidas por la comunidad en general y por la clase política en particular. Es prioritario trabajar por la infancia entre las comunidades, los dirigentes, los empresarios y todos los sectores sociales, para visibilizar a los niños y las niñas como seres en desarrollo, que necesitan la solidaridad y el apoyo de la comunidad adulta.

La ciudadanía y la participación están potencialmente en todas las actividades que realizan los niños, el papel del adulto es convertirlas en acto. El trabajo más grande es cambiar el imaginario que los adultos tienen de los niños y las niñas, es importante visibilizarlos aún más, reconocerlos desde su subjetividad como fuente de riqueza humana. Tonucci (1991) planteaba que si existieran más niños en las calles las ciudades serían más tranquilas, porque los adultos en su condición moderan más sus acciones ante la presencia de los chicos. Así, el mundo de hoy requiere, por un lado, pensar nuevas formas de ciudadanía para la construcción de sociedades más incluyentes, y por otro lado, atender las formas de socialización de los más pequeños para construir sociedades más democráticas y más humanizantes.

Panorama

pp. 102-119।

Volumen 101

Número 18 I

Enero-junio I

2016 I

Negar el derecho a participar realmente y estar informados y expresarse de manera libre sobre lo que los afecta, así como desconocer su capacidad para aportar ideas, va en contra de los principios de una formación democrática y genera en ellos desconfianza, indiferencia, incomprensión y falta de compromiso con la comunidad en la que habitan, con lo cual se limitan las condiciones que les permitirían reconocer y apropiarse de elementos
Se puede señalar que existe una relación entre el modo como conciben su ciudadanía las madres comunitarias y la formación que brindan a los niños y las niñas. Así, es preciso reconocer los aportes que ofrecen para la construcción en ciudadanía en la primera infancia, desde la perspectiva de la ética del cuidado, porque posibilitan la construcción de una "cultura de la ternura" (Singer y De Haan, 2008), a partir de la confianza, el afecto y el buen trato que les brindan a sus pequeños, que favorecen el desarrollo de elementos para el reconocimiento y cuidado por el otro (Nussbaum, 2010).

Las madres comunitarias ayudan al cultivo de las emociones morales, como la compasión, la empatía, la reciprocidad, la reflexión sensible sobre el sufrimiento de otros, el sentimiento de vulnerabilidad y el ideal de una necesidad mutua, que son del todo significativos para sociedades más democráticas y humanas.

\section{RECOMENDACIONES}

1. Si bien la formación dada por el SENA ha logrado tener un impacto positivo en la comunidad de madres comunitarias, es preciso seguir apoyando sus procesos de formación para que lo adquirido en el programa Técnico en Atención Integral a la Primera Infancia tenga mayor impacto con los niños y las niñas. Se recomienda que se valide el certificado que reciben las madres comunitarias en los centros universitarios para que puedan realizar estudios de profesionalización. Este reconocimiento, como el de otros derechos, debe ser una prioridad por parte del Estado, pues su incumplimiento afecta la labor que realizan con los niños y las niñas.

2. Es necesario construir una cultura que comprenda al niño y a la niña como sujetos ciudadanos: en cada una de las instituciones que trabaja con la primera infancia deben conocerse la Convención sobre los Derechos del Niño. Esta debe estar publicada en lugares visibles del jardín y ser socializada para que toda la comunidad la conozca, en especial los mismos niños, con quienes debe abrirse espacios de diálogo y reflexión al respecto.

3. Se deben favorecer espacios para que los niños y las niñas tengan una mayor posibilidad de dialogar y escucharse entre pares, que los niños se oigan e interactúen, porque entre ellos se afirman y niegan sentencias, estas 
se polemizan y desarrollan un aprendizaje colaborativo desde los niños y las niñas.

4. La formación en ciudadanía en la primera infancia tiene implicaciones que trascienden el espacio del hogar comunitario en cuanto todos los niños deben vivir en una ciudad tranquila y segura, por lo que esta debe repensarse en pro de los niños y las niñas, si se quiere hacer de ellos ciudadanos, la sociedad debe hacérseles sentir que lo son, lo cual no se logra solo desde el cumplimiento de sus derechos. La ciudad debe ser el hogar público, y lograr esto permite que el niño quiera adherirse a los proyectos de la ciudad, porque sabe que esta piensa en él y así se forja un sentido de pertenencia, el cual es una condición de la virtud moral de la civilidad (Cortina, 1997).

Sin espacios de participación para la infancia, donde los niños no tengan la oportunidad de dialogar, comunicarse, de jugar, recrearse, plantear sus inconformidades y gustos, de vivir de manera segura, se desquebraja su autonomía y se desfavorece su autoestima: "La ciudad ha perdido a los niños, y los niños han perdido la ciudad" (Tonucci, 1991).

5. Las expresiones de los niños tienen que ser visibilizadas, los jardines deberían tener espacios donde se muestren sus preguntas, necesidades, temores, gustos, sueños, alegrías, qué los hace llorar, suspirar, sonreír. Ello debe ser leído por todos, en especial por los padres de familia, para que vean las capacidades argumentativas, lógicas, de inferencia que los niños pueden hacer, sus recursos artísticos, lo cual genera un cambio de mirada hacia los niños y los padres empiezan a cuestionarse.

6. La formación ciudadana en la primera infancia debe estar direccionada hacia un proyecto de vida para los niños y las niñas. Por ello, desde los hogares comunitarios y jardines se debe cultivar en los pequeños la idea de este proyecto para fortalecer su autoestima, porque se les concientiza que han llegado a la vida por algo, para construir y hacer cosas, con lo cual pueden aportar a sus entornos, y esto les permite estar más atentos de lo que pueden llegar a desarrollar.

Es una prioridad inculcar "los sentidos" de lo que se hace o realiza en y con los niños. Si los griegos se preocupaban tanto por el cultivo de las virtudes morales era porque su fin y sentido era alcanzar la felicidad.

7. Una de las dimensiones de la ciudadanía es la ciudadanía pluricultural, por tanto, en los hogares comunitarios deben planearse más actividades de reconocimiento del otro desde las tradiciones y costumbres de los diferentes grupos étnicos. Ello en virtud de atender de manera eficaz una educación desde la perspectiva diferencial.

8. Debe procurarse fortalecer una formación moral desde la infancia. Los niños formulan juicios morales de acuerdo con sus realidades sociales (al parecer siempre estamos trabajando en la perspectiva de qué hacer para los niños y no con los niños).

9. Es preciso una nueva mirada de la relación niñoagente educativo. Las relaciones docente, cuidador, adulto-niño-niña han sido históricamente asimétricas, por ello, deben resignificarse las nociones de poder $y$ autoridad. Por ejemplo, los adultos "tienen derecho" a usar el teléfono, los niños no; a hablar mal del otro, a no ser colaborativo, a vivir de mal genio, a no cumplir lo prometido, a no respetar la palabra. A la percepción de los pequeños esto puede resultar autoritario, de manera que se debe ser consecuente con lo que se plantea si se quieren favorecer los ejercicios de participación y ciudadanía. Por ello, la necesidad de construir un proyecto transversal a todas y cada una de las actividades que se dan en los hogares comunitarios, en un contexto que forje relaciones más simétricas.

10. Un tema importante para la formación ciudadana, como lo hicieron notar las madres comunitarias, es el cuidado de sí, porque permite:

A. Reconocernos como seres humanos (habría sociedades más equitativas).

B. El cuidado del otro y de lo otro (condición fundamental de la ciudadanía).

El cuerpo es el lugar que habitamos y somos, a través del cual nos revelamos a los demás, pero aparte de propiciar el deporte y las expresiones artísticas para el cultivo de la corporeidad, qué bueno llevar a los niños a tener experiencias internas que los concientice de ellos. Además, idear sesiones de silencio para escuchar su propia voz, hacerles reconocer que los humanos sentimos rabia

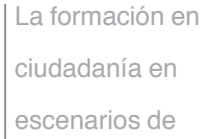

educación inicial: una experiencia con madres comunitarias 
Arly Adriana

Rodríguez

Huesa I

Rita Flórez

Romero I

Diana Gómez

Muñoz I pero que debemos saber manejarla, que lloramos, tenemos miedo, nos aburrimos, dudamos... En últimas, es volver sobre procesos que permitan el cultivo de estas personitas como seres humanos.

11. El cultivo del juego es fundamental para hablar de ciudadanía en la infancia. Los niños deben jugar y jugar porque es allí donde aprenden verdaderamente a cómo ser ciudadano en cuanto están en una constante socialización con los otros. Por lo cual es fundamental dejar que los niños jueguen, tener varios espacios para el tiempo libre, que permitan el encuentro de sí y de los otros.

12. La ciudadanía y la participación están potencialmente en todas las actividades que realizan los niños, y el papel del adulto es convertirla en acto. El trabajo más grande es cambiar el imaginario que tenemos hacia los niños, es importante visibilizarlos aún más.

El reto es construir una sociedad donde los pequeños tengan voz y rostro. La historia de la sociedad colombiana se ha construido a partir de una suma de sujetos que no fueron formados como ciudadanos, en la que se han vulnerado e irrespetado los derechos propios y los derechos de los otros, en cuyas almas no se trazó la idea de un proyecto de vida ni como sujetos particulares ni como país, por ello la infortunada realidad en la que estamos sumergidos, de manera que resulta relevante formar en estos procesos para una sociedad más justa, libre y feliz, desde una lógica no contemplada por la modernidad: la lógica de la infancia.

\section{REFERENCIAS}

1. Acosta, A. y Pineda, N. (2007). Ciudad y participación infantil. En Y. Corona Caraveo y M. E. Linares Pontón (coords.), Participación infantily juvenil en América Latina (pp. 147-177). México: Universidad Autónoma de México.

Panoramal

pp. 102-119

Volumen 10

Número 18

Enero-junio I

2016 |

3. Apud, A. (s. f.). Participación infantil. Recuperado de http://www.sename.cl/wsename/ otros/unicef.pdf
4. Colombia, Congreso de la República. Ley 1098 de 2006 (8 noviembre 2006).

5. Comisión Intersectorial para la Atención Integral a la Primera Infancia (2012).

Lineamiento técnico de participación y e jercicio de la ciudadania en la primera infancia. Recuperado de http://www.deceroasiempre.gov.co/ QuienesSomos/Documents/6.De-Participaciony-ejercicio-Ciudadania-en-la-Primera-Infancia. pdf

6. Comisión Intersectorial para la Atención Integral a la Primera Infancia (2014). Estrategia de atención integral a la primera infancia. Bogotá.

7. Comité de los Derechos del Niño. Observación General N.o 7 (2005). Recuperado de http:// www.unicef.cl/web/informes/derechos_nino/07. pdf

8. Consejo Nacional de Política Económica Social, Departamento Nacional de Planeación. Conpes 109 (3 diciembre 2007)

9. Conversación con Roger Hart (s. f). Recuperado de http://www.colombiaaprende.edu.co/html/ mediateca/1607/articles-169196_archivo.pdf

10. Cortina, A. (1997). Ciudadanos del mundo: hacia una teoría de la ciudadanía. Madrid: Alianza.

11. Elshtain, J. B. y Decosse, D. E. (2006). Public man, private woman: Women in social and political thought. Princeton: Princeton University Press.

12. Estrada, M. V., Madrid-Malo, E. y Gil, L. M. (2000). La participación está en juego. Bogotá: Fundación Antonio Restrepo Barco, Unicef.

13. Flórez, R. y Torrado, madre comunitaria (2013). Primera infancia, lenguajes e inclusión social: una mirada desde la investigación. Bogotá: Universidad Santo Tomás, Universidad Nacional de Colombia.

14. Garzón Rodríguez, J., Pineda Báez, N. y Acosta Ayerbe, A. (2004). Informe sobre la revisión de algunas experiencias de participación infantil. Bogotá: Cinde.

15. Gilligan, C. (1985). La moral y la teoría: psicología del desarrollo femenino. México: Fondo de Cultura Económica.

16. Hart, R. A. (1993). La participación de los niños: de una participación simbólica a una participación auténtica. Ensayos Innocenti, 4. Unicef. Recuperado de https://www.unicef-irc.org/ publications/pdf/ie_participation_spa.pdf

17. Hart, R. A. (2001). La participación de los niños en el desarrollo sostenible. Barcelona, Madrid: Unicef, PAU Education. 
18. Hart, R. A. (2013). Children's participation: The theory and practice of involving young citizens in community development and environmental care. Londres, Nueva York: Earthscan.

19. Kant, I. (2006). La facultad de juzgar. Venezuela: Monte Avila.

20. Lago, J. C. (2006). Redescribiendo la comunidad de investigación: pensamiento complejo y exclusión social. Madrid: Ediciones de la Torre.

21. Lipman, M. (1991). Thinking in education Cambridge: Cambridge UniversityPress.

22. Lipman, M. (1998). Pensamiento complejo y educación. Madrid: Ediciones de la Torre.

23. Memorias lanzamientos estrategia nacional (2011). Recuperado de http://www.deceroasiempre.gov.co/Prensa/Documents/ Lanzamiento-estrategia-nacional.pdf

24. Ministerio de Educación Nacional (2009). Desarrollo infantil y competencias en la primera infancia. Recuperado de http://www. mineducacion.gov.co/cvn/1665/articles-210305 archivo_pdf.pdf

25. Mouffe, C. (1992). Feminism, citizenship, and radical democratic politics. En J. Butler y J.W. Scott (ed.), Feminists theorize the political. Nueva York: Routledge.

26. Mouffe, C. (1999). El retorno de lo politico: comunidad, ciudadania, pluralismo, democracia radical. Barcelona, Paidós.

27. Mouffe, C. y Moreno, H. (1993). Feminismo, ciudadanía y política democrática radical. Debate Feminista, 7, 3-22.

28. Nucci, L. y Weber, E. K. (1995). Social interactions in the home and the development of young children's conceptions of the personal. Child Development, 66(5), 1438-1452.

29. Nussbaum, M. (2010). Sin fines de lucro:por qué la democracia necesita de las humanidades. Buenos Aires: Katz.

30. Pineda, D. (2004). El ABC de filosofía para niños. Barcelona: Beta.

31. Palma, E. y Pizarro, C. (1997). Niñez y democracia. Bogotá: Unicef.

32. Ruddick, S. (1989). Maternal thinking. Londres: Verso.
33. Singer, E. y De Haan, D. (2008). Los procesos de socialización en la primera infancia: juegos, conflictos y reconciliación en los centros infantiles. Ámsterdam: SWP.

34. Tonucci, F. (1991). La ciudad de los niños. Buenos Aires: Losada.

35. Torrado, M. E. y Reyes, M. E. (2006). Bases para la formulación de un plan nacional para el desarrollo de la primera infancia. Revista Infancia Adolescencia y Familia, 1(1), 15-38.

36. Torrado, M. C. (2009). Retos para las politicas de primera infancia. Bogotá: Universidad Nacional de Colombia.

37. Winnicott, D. W. (1975). El proceso de maduración en el niño. Barcelona: LAIA.
La formación en
ciudadanía en
escenarios de

educación inicial: una experiencia

con madres

comunitarias 